\title{
Coupled Model of Artificial Neural Network and Grey Model for Tendency Prediction of Labor Turnover
}

\author{
Yueru Ma and Lijun Peng \\ Business School, Central South University, Changsha, Hunan 410083, China \\ Correspondence should be addressed to Lijun Peng; penlijincaozhi@sina.com
}

Received 20 December 2013; Revised 9 March 2014; Accepted 11 March 2014; Published 15 April 2014

Academic Editor: Gongnan Xie

Copyright (C) 2014 Y. Ma and L. Peng. This is an open access article distributed under the Creative Commons Attribution License, which permits unrestricted use, distribution, and reproduction in any medium, provided the original work is properly cited.

\begin{abstract}
The tendency of labor turnover in the Chinese enterprise shows the characteristics of seasonal fluctuations and irregular distribution of various factors, especially the Chinese traditional social and cultural characteristics. In this paper, we present a coupled model for the tendency prediction of labor turnover. In the model, a time series of tendency prediction of labor turnover was expressed as trend item and its random item. Trend item of tendency prediction of labor turnover is predicted using Grey theory. Random item of trend item is calculated by artificial neural network model (ANN). A case study is presented by the data of 24 months in a Chinese matured enterprise. The model uses the advantages of "accumulative generation" of a Grey prediction method, which weakens the original sequence of random disturbance factors and increases the regularity of data. It also takes full advantage of the ANN model approximation performance, which has a capacity to solve economic problems rapidly, describes the nonlinear relationship easily, and avoids the defects of Grey theory.
\end{abstract}

\section{Introduction}

New classical economists are sticking to a fundamental assumption: the social and economic phenomenon can be predicted. The mathematical model can predict the long-term trend of the economic problem development and help rational decision-makers to achieve rapid development [1-3]. Due to the effect of social and economic cyclical, demographic characteristics and the causes of work-life balance, the human resource is one of the most unstable productive factors in enterprises $[4,5]$. The mathematical model is needed to establish a set of index systems and analysis method. By the model we can find the inner logic and relatively stable structure of employee turnover, analyze the trend of human resource flow, identify potential crisis, shorten the difference time between searching and using the labor, reasonable plan, and control the strategy combination of human resource, as well as deposit the completion of sustainable productive tasks in enterprises [6-8]. Many models and technologies for forecasting the trends of economic problem, such as Grey model (GM), artificial neural network (ANN), time series analysis, and the combination model, were developed in the past years. However, the predictive models do not meet the practical conditions with the characteristics of random and complex, nonlinear and volatility, as well as localization and contextualized $[9,10]$. In this paper, the combinative model of grey model and artificial neural network $(\mathrm{CM})$ is used to handle the Chinese enterprise employee flowing data and predict the current trend of Chinese enterprise employee.

In this work, the following aspects will be explained and analyzed: (1) designing concrete model to provide key indicators for economic data with characteristic of time series, which can be used to track the trend, change, and mutant of economic phenomenon. The model can explain the relationship between the current and previous data in economic problems and focus on the change in a specific period; (2) analyzing the annual turnover rate and the details and trends of annual turnover flow, and identifying or expecting the shortage or excess of employee number by the application of the model. Thus, it can provide the basis of hiring, allocating, or dismissing employee for rational decision makers. The rational decision makers can better control cost and comply with budget constraints.

The core of these methods is to establish the appropriate forecasting model. Because of the complexity of time series and the nonlinear characteristics of the tendency prediction 
system, it is difficult to establish the ideal forecast model. In addition, the given uncertain tendency prediction is a chaotic system, so the pursuit of the prediction of longterm development and evolution of tendency prediction is unrealistic, but the prediction of short-term behavior of their degree is possible.

Therefore, a short-term ideal forecast model should meet two conditions: (i) a theory that can predict time series, (ii) the advantages of a theory that are modeling of nonlinear functions, and good performance in noisy environments. However, it is difficult to find a theory to meet the two conditions. It is possible to establish a model based on the two theories. Grey theory has been successfully used to predict time series. ANN, Random Forest, Support Vector Machine, BP Neural Network, Uncertainty Measurement, and Bayes methods are also used to solve nonlinear problems [11-16]. In the work, the ANN was selected to fit the nonlinear performance of the labor turnover, since the major advantages of an artificial neural network (ANN) are $[12,17]$ fast training, modeling of nonlinear functions, and good performance in noisy environments that gives enough data.

We present a coupled model of Grey model and artificial neural network for predicting tendency prediction of labor turnover [18]. In this model, a time series of tendency prediction of labor turnover was expressed as trend item and its random item. The trend item of tendency prediction of labor turnover is predicted with Grey theory. Random item of trend item is calculated by ANN. The model uses the advantages of "accumulative generation" of a Grey prediction method, which weakens the original sequence of random disturbance factors and increases the regularity of data. It also takes full advantage of the ANN model approximation performance because it has fast solving speed and can describe the nonlinear relationship easily and avoid the defects of Grey theory [19-22].

\section{Methodology}

2.1. Brief Mathematical Explanation of an Artificial Neural Network. Neural network models in artificial intelligence are usually referred to as artificial neural networks (ANNs) [12, 17]; these are essentially simple mathematical models defining a function or a distribution, but sometimes models are also intimately associated with a particular learning algorithm or learning rule. A common use of the phrase ANN model really means the definition of a class of such functions (where members of the class are obtained by varying parameters, connection weights, or specifics of the architecture such as the number of neurons or their connectivity).

Models. The models presented in this section appear fairly difficult mathematically. However, they eventually boil down to just multiplication and addition. The use of matrices and vectors simplifies the notation but is not absolutely required for this application.

Neuron Model. A model of a neuron has three basic parts: input weights, a summer, and an output function. The input weights scale values used as inputs to the neuron, the summer

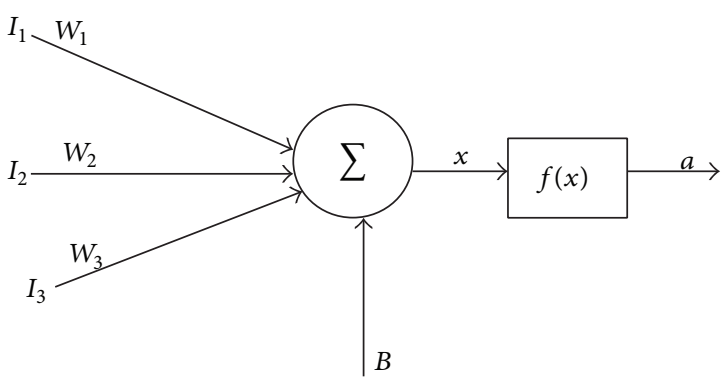

Figure 1: Neuron model.

adds all the scaled values together, and the output function produces the final output of the neuron. One additional input, known as the bias, is often added to the system. If a bias is used, it can be represented by a weight with a constant input of one. This description is laid out visually in Figure 1.

$I_{1}, I_{2}$, and $I_{3}$ are the inputs, $W_{1}, W_{2}$, and $W_{3}$ are the weights, $B$ is the bias, $x$ is an intermediate output, and a is the final output. The equation for $a$ is given by

$$
a=f\left(W_{1} I_{1}+W_{2} I_{2}+W_{3} I_{3}+B\right),
$$

where $f$ could be of any function. Most often, $f$ is the sign of the argument (i.e., 1 if the argument is positive and -1 if the argument is negative), linear (i.e., the output is simply the input times some constant factor), or some complex curve used in function matching (not needed here). For this model we will use the first case where $f$ is the sign of the argument for two reasons: it closely matches the "all or nothing" property seen in biological neurons and it is fairly easy to be implemented.

When artificial neurons are implemented, vectors are commonly used to represent the inputs and the weights so the first of two brief reviews of linear algebra is appropriate here. The dot product of two vectors $\vec{x}=\left(x_{1}, x_{2}, \ldots, x_{n}\right)$ and $\vec{y}=\left(y_{1}, y_{2}, \ldots, y_{n}\right)$ is given by

$$
\vec{x} \cdot \vec{y}=x_{1} y_{1}+x_{2} y_{2}+\cdots+x_{n} y_{n} .
$$

Using this notation the output is simplified to

$$
a=f(\vec{W} \cdot \vec{I}+B)
$$

where all the inputs are contained in $\vec{I}$ and all the weights are contained in $\vec{W}$.

Neuron Layer. In a neuron layer each input is tied to every neuron and each neuron produces its own output. This can be represented mathematically by the following series of equations:

$$
\begin{aligned}
& a_{1}=f_{1}\left(\vec{W}_{1} \cdot \vec{I}+B_{1}\right), \\
& a_{2}=f_{2}\left(\vec{W}_{2} \cdot \vec{I}+B_{2}\right), \\
& a_{3}=f_{3}\left(\vec{W}_{3} \cdot \vec{I}+B_{3}\right)
\end{aligned}
$$


Representing the input vector and the biases as one column matrices, we can simplify the above notation to

$$
\vec{a}=f(W \cdot I+B)
$$

which is the final form of the mathematical representation of one layer of artificial neurons.

Neural Network. A neural network is simply a collection of neuron layers where the output of each previous layer becomes the input to the next layer. So, for example, the inputs to layer two are the outputs of layer one. In this exercise we are keeping it relatively simple by not having feedback (i.e., output from layer $n$ being input for some previous layer). To mathematically represent the neural network we only have to chain together the equations. The finished equation for the three-layer network in this equation is given by

$$
a=f\left(W_{3} \cdot f\left(W_{2} \cdot f\left(W_{1} \cdot \vec{I}+\vec{B}_{1}\right)+\vec{B}_{2}\right)+\vec{B}_{3}\right) .
$$

2.2. GM-ANN Model. In this model, a time series of tendency prediction of labor turnover was expressed as trend item and its random item. Trend item of deformation, which is related by time, is predicted with Grey theory [22]. Random item of trend item, which is a complex nonlinear sequence, is calculated by ANN [18]. We resolve a time series of tendency prediction of labor turnover $W(t)$ as

$$
W(t)=Y(X(t), t),
$$

where series of trend item $X(t)$ can be expressed as the grey model and series of random item of trend item $Y(X(t), t)$ can be expressed as the ANN model. For time series $X(t)$, after one accumulative generation, we obtain

$$
X^{(1)}(t)=\sum_{k=1}^{t} X(k), \quad(t=1,2, \ldots, M)
$$

and series $X^{(1)}(t)$ satisfies GM $(1,1)$ model

$$
\frac{d x^{(1)}(t)}{d t}+a x^{(1)}(t)=b .
$$

We can know easily that

$$
\begin{gathered}
x(t)=x^{(1)}(t)-x^{(1)}(t-1), \quad t=1,2, \ldots, M . \\
X^{(1)}(t)=\left(X^{(1)}(0)-\frac{b}{a}\right) e^{-a t}+\frac{b}{a} .
\end{gathered}
$$

Suppose series of random item $Y(X(t), t)$ satisfied ANN model. The architecture of a basic ANN model is shown in Figure 2. The model has three layers: input, summation, and output with the weighted connections between the inputs and the summation layers.

The model used for the present study has two input nodes (spatial coordinates), $j$ pattern two $(1+1)$ summation nodes and one output nodes. The function of the input layer is to pass forward the activity patterns presented to the network to all nodes in the pattern layer. The nodes in the pattern layer

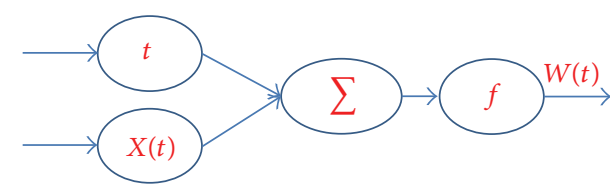

Figure 2: Architecture of ANN.

TABLe 1: Data of turnover of employees from the Chinese A enterprises in 2011.

\begin{tabular}{lcccccccccccc}
\hline & \multicolumn{11}{c}{ Month $(t)$} \\
& 1 & 2 & 3 & 4 & 5 & 6 & 7 & 8 & 9 & 10 & 11 & 12 \\
\hline (1) Entry, $x(t)$ & 3 & 5 & 8 & 8 & 4 & 1 & 2 & 3 & 3 & 1 & 2 & 0 \\
(2) Exit, $y(t)$ & 0 & 3 & 21 & 9 & 17 & 9 & 6 & 21 & 11 & 6 & 3 & 8 \\
\hline
\end{tabular}

perform a nonlinear transformation of the input patterns. When a new vector $X$ is entered into the network, it is subtracted from the stored weight vector representing each cluster centre [23]. Based on the above theories in this paper, we produce a program of ANN model using MATLAB 2011a. Applied steps of the program are as follows: (i) according to the measured data of tendency prediction of labor turnover, the predicted sequence is obtained using the established GM $(1,1)$ model part of the program; (ii) In the ANN model, the predicted sequence and time can be selected as input variables, the measured data of tendency prediction of labor turnover can be selected as expected variables. The ANN model is trained to predict the tendency prediction of labor turnover according to the time and Grey prediction. As the smooth factor affects network performance, it is needed to keep trying to determine the best value; (iii) if we want to know the the measured data of tendency prediction of labor turnover in a future month, we will get the accurate predicted value after only entering the required time into the wellestablished ANN model. Then it will serve scientific and reasonable decision makers.

\section{Establishing Models and Discussions}

This model is based on data from the Chinese enterprise A. Enterprise A is relatively matured and stable economies. Its business develops steadily in the process of the rapid expansion of the Chinese economy. This study used two years of data of enterprise A (specific as shown in Tables 1 and 2 ). The data is a mapping of staff flow trend in the Chinese enterprises in recent years. The data follow the two aspects of the law: a law of time series data is followed; and the nonlinear relationship between the data. On the basis of GM $(1,1)$ models (i.e., model 1, model 2, model 3, and model 4), the optimized models based on ANN of GM $(1,1)$ (i.e., coupling model 1 , coupling model 2 , coupling model 3 , and coupling model 4) are obtained.

The GM model of entry number for employees in 2011, the GM model of exit number for employees in 2011, the GM 


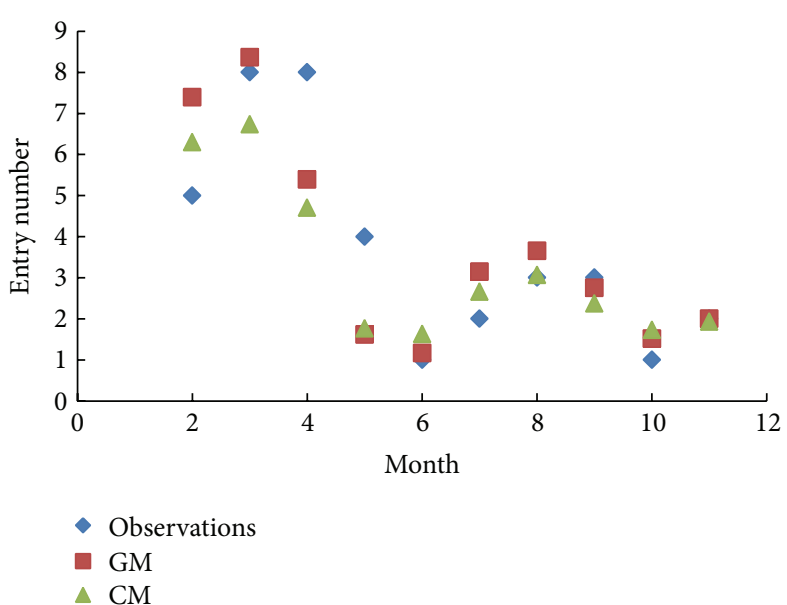

(a) Comparisons of entry number in 2011

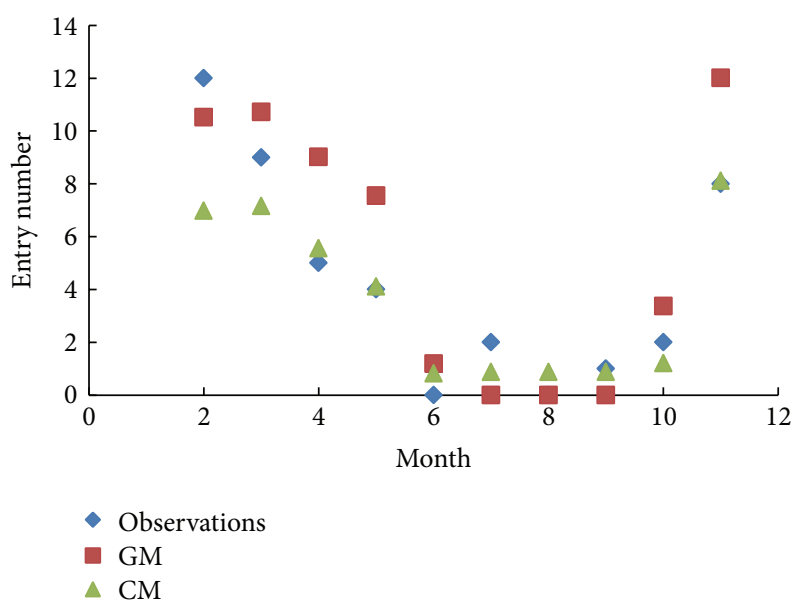

(c) Comparisons of entry number in 2012

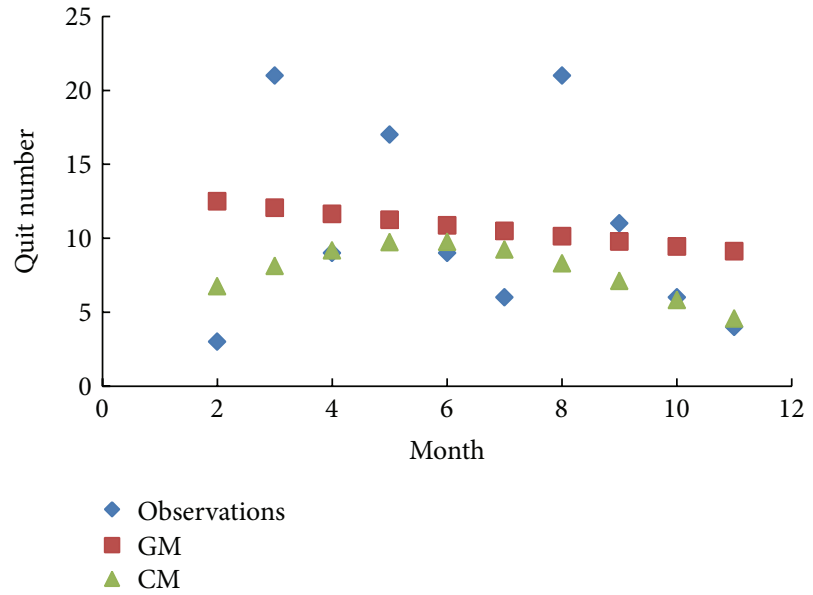

(b) Comparisons of quit number in 2011

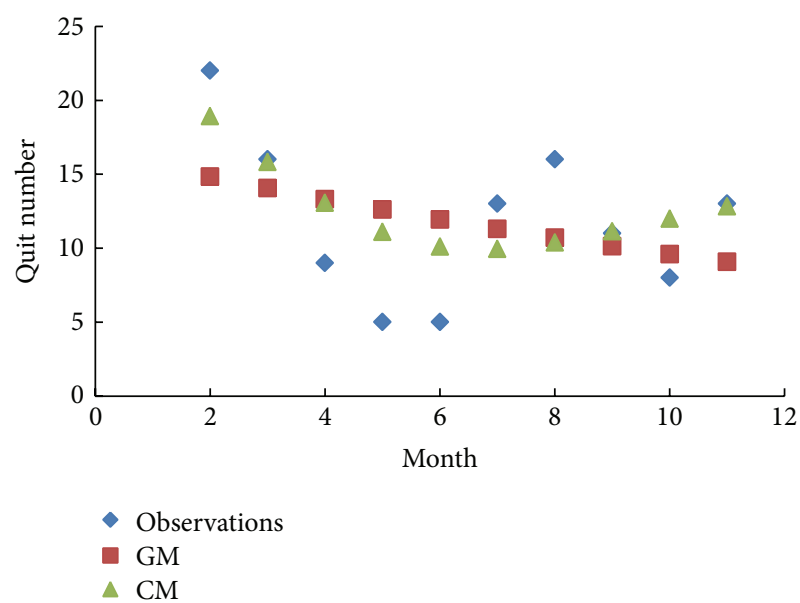

(d) Comparisons of quit number in 2012

Figure 3: Results and comparisons of entry and quit number.

model of entry number for employees in 2012, and the GM model of exit number for employees in 2012 were obtained as

$$
\begin{aligned}
& x(t+1)=-158.563366 e^{-0.015188 t}+160.710719 \\
& x(t+1)=-362.298701 e^{-0.035088 t}+362.298701 \\
& x(t+1)=21.413473 e^{0.134079 t}-14.761375 \\
& x(t+1)=-279.593023 e^{-0.054556 t}+279.593023
\end{aligned}
$$

GM often only considers time series, which is used to increase exponential time betrayal. Apparently the reality of the economic data is more complex, although the time series and nonlinear data can be considered together. Considering the complexity of the Chinese employee turnover trend, it is necessary for time series and nonlinear to fit the method in model building. Therefore, according to the GM $(1,1)$ time series model is established, and the ANN was used to modify the GM $(1,1)$ models.

The calculated results of 2-11 months with GM, CM, and observations are shown in Figure 3. The predicted result of
TABle 2: Data of turnover of employees from the Chinese A enterprises in 2012.

$$
\text { Month }(t)
$$

\begin{tabular}{lcccccccccccc} 
& 1 & 2 & 3 & 4 & 5 & 6 & 7 & 8 & 9 & 10 & 11 & 12 \\
\hline (1) Entry, $x(t)$ & 6 & 12 & 9 & 5 & 4 & 0 & 2 & 0 & 1 & 2 & 8 & 7
\end{tabular}

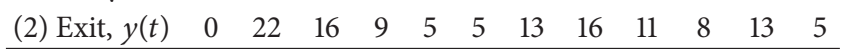

TABle 3: Predicted results of December.

\begin{tabular}{lcccc}
\hline & $\begin{array}{c}\text { Entry in } \\
2011\end{array}$ & $\begin{array}{c}\text { Quit in } \\
2011\end{array}$ & $\begin{array}{c}\text { Entry in } \\
2012\end{array}$ & $\begin{array}{c}\text { Quit in } \\
2012\end{array}$ \\
\hline Observation & 0 & 8 & 7 & 5 \\
GM & 1.17815 & 8.79513 & 1.35858 & 8.60282 \\
Error & -1.17815 & -0.79513 & 5.64142 & -3.60282 \\
CM & 1 & 7.5691932 & 7.8233 & 5.83 \\
Error & -1 & 0.4308068 & -0.8233 & -0.83 \\
\hline
\end{tabular}

December is listed in Table 3, and the errors were discussed. Figure 3(a) shows the results of CM for months 2, 4, 5, 7, 
9, 10, and 11 that are consistent with observations, and the errors are smaller than the results of GM. The errors of results for months 3 and 6 by GM are smaller than that of CM. Figure 3(b) shows that results of CM for months 1, 2, and 9 are not well consistent with observations, but the average errors are smaller than the results of GM. Figure 3(c) shows that results of CM for months 3, 4, 5, 6, 7, 9, 10, and 11 are well consistent with observations, and the errors are smaller than the results of GM. The errors of results for months 2 and 8 by GM are smaller than that of CM. Figure 3(d) shows that results of CM for months 2, 3, 4, 5, 6, 9, and 11 are well consistent with observations, and the errors are smaller than the results of GM. The errors of results for months 2, 7, 8, and 10 by GM are smaller than that of CM. Table 3 shows that errors of predicted results for entry in 2011, quit in 2011, and quit in 2012 by CM are smaller than that of GM. The CM is failed to predict the entry in 2012. From above analysis, it can be clearly seen that the average accuracy of CM is smaller than that of GM, which has demonstrated that the proposed CM can analyze the tendency prediction of labor turnover, though there still exist some errors in CM.

\section{Conclusions}

In the present work, a coupling model with use of the GM and ANN is presented. The aim of the present study was to develop a reliable model for the problem of tendency prediction of labor turnover. According to the existing data of the enterprise, the predicted sequence is obtained using the established GM $(1,1)$ model in that part of the program. In part of general regression neural network model, predicted sequence and time are input variables; the existing data is expected variables. The ANN model is trained to predict tendency prediction of labor turnover. The model is applied to the data of 24 months in a Chinese matured enterprise. It shows that the approximation error of the proposed model is small. Results show that the proposed model has a capacity to solve economic problems rapidly, which can describe the nonlinear relationship easily and can avoid the defects of Grey theory.

\section{Conflict of Interests}

The authors declare that there is no conflict of interests regarding the publication of this paper.

\section{References}

[1] A. Denvir and F. McMahon, "Labour turnover in London hotels and the cost effectiveness of preventative measures," International Journal of Hospitality Management, vol. 11, no. 2, pp. 143-154, 1992.

[2] L. A. Peletier and J. Gabrielsson, "Nonlinear turnover models for systems with physiological limits," European Journal of Pharmaceutical Sciences, vol. 37, no. 1, pp. 11-26, 2009.

[3] Y. Zhang, T. Gong, and Y. Zhang, "A forecasting model for employees/ exceptional turnover based on calamities Grey Prediction Theory," Mathematics in Practice and Theory, vol. 20, article 9, 2008.
[4] P. C. Pendharkar, J. A. Rodger, and K. Wibowo, "Artificial neural network based data mining application for learning, discovering and forecasting human resources requirement in Pennsylvania Health Care Industry," Pennsylvania Journal of Business and Economics, vol. 7, no. 1, pp. 91-111, 2000.

[5] A.-L. Balduck, A. Prinzie, and M. Buelens, "The effectiveness of coach turnover and the effect on home team advantage, team quality and team ranking," Journal of Applied Statistics, vol. 37, no. 4, pp. 679-689, 2010.

[6] R. S. Sexton, S. McMurtrey, J. O. Michalopoulos, and A. M. Smith, "Employee turnover: a neural network solution," Computers and Operations Research, vol. 32, no. 10, pp. 26352651, 2005.

[7] A. R. AlBattat, A. P. M. Som, and A. S. Helalat, "Overcome staff turnover in the Hospitality Industry using Mobley Model," International Journal of Learning and Development, vol. 3, no. 6, pp. 64-71, 2013.

[8] C.-T. Lin, Y.-H. Wang, and W.-R. Lin, "Information effect of top executive turnover: testing hybrid grey-market model," Journal of Grey System, vol. 20, no. 1, pp. 53-64, 2008.

[9] N. M. Abbas, "Neural network solution to economic forecasting," in Proceedings of the International Conference on Business Intelligence and Knowledge Economy, Amman, Jordan, April 2012.

[10] A. Subasi and E. ilgün, "Economic variable forecasting using artificial neural network: A Case Study in Turkey," in Proceedings of the 1st International Symposium on Sustainable Development, Sarajevo, Bosnia and Herzegovina, June 2009.

[11] J. L. Deng, A Course on Grey System Theory, Huazhong University of Science and Technology Press, Wuhan, China, 1990.

[12] R. J. Schalkoff, Artificial Neural Networks, McGraw-Hill Higher Education, 1997.

[13] G. J. Burghouts, "Soft-assignment random-forest with an application to discriminative representation of human actions in videos," International Journal of Pattern Recognition and Artificial Intelligence, vol. 27, no. 4, 2013.

[14] J. A. K. Suykens and J. Vandewalle, "Least squares support vector machine classifiers," Neural Processing Letters, vol. 9, no. 3, pp. 293-300, 1999.

[15] D. Zhou, "Optimization modeling for GM (1,1) Model Based on BP Neural Network," International Journal of Computer Network and Information Security, vol. 4, no. 1, article 24, 2012.

[16] I. Dialsingh, "Large-scale inference: empirical Bayes methods for estimation, testing, and prediction," Journal of Applied Statistics, vol. 39, no. 10, pp. 2305-2305, 2012.

[17] V. Cherkassky, J. H. Friedman, and H. Wechsler, From Statistics to Neural Networks: Theory and Pattern Recognition Applications, Springer, 2012.

[18] J. Li, A. Yang, and W. Dai, "Modeling mechanism of grey neural network and its application," in Proceedings of the IEEE International Conference on Grey Systems and Intelligent Services (GSIS '07), pp. 404-408, November 2007.

[19] L. Dong and X. Li, "An application of grey-general regression neural network for predicting landslide deformation of Dahu Mine in China," Advanced Science Letters, vol. 6, no. 1, pp. 577581, 2012.

[20] H. Duan, B. Shen, and S. Mo, "Study on combined predictable model of Gray GM $(1, \mathrm{~N})$ self-memory and neural network," Journal of Water Resources and Water Engineering, vol. 5, article 23, 2010. 
[21] P. Xiong, Y. Dang, X. Wu, and X. Li, "Combined model based on optimized multi-variable grey model and multiple linear regression," Journal of Systems Engineering and Electronics, vol. 22, no. 4, pp. 615-620, 2011.

[22] L. Dong and X. Li, "Comprehensive models for evaluating rockmass stability based on statistical comparisons of multiple classifiers," Mathematical Problems in Engineering, vol. 2013, Article ID 395096, 9 pages, 2013.

[23] Yueya, L. Mingjie, and Z. Y. Shi, "Turnover of passenger traffic prediction for civil aviation based on GM $(1,1)$ model," Journal of Civil Aviation Flight University of China, vol. 5, article 9, 2011. 


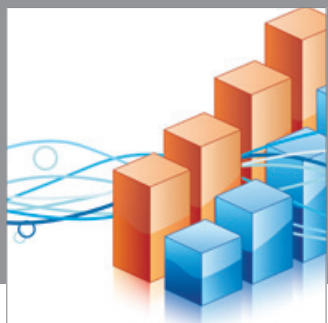

Advances in

Operations Research

mansans

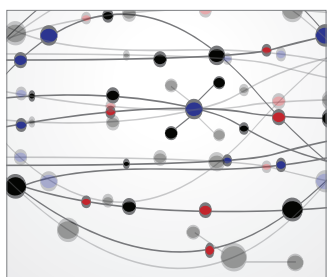

The Scientific World Journal
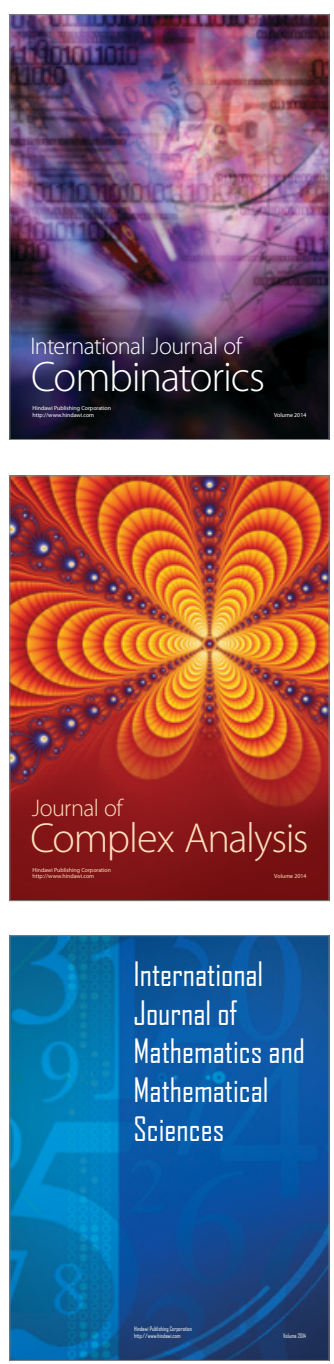
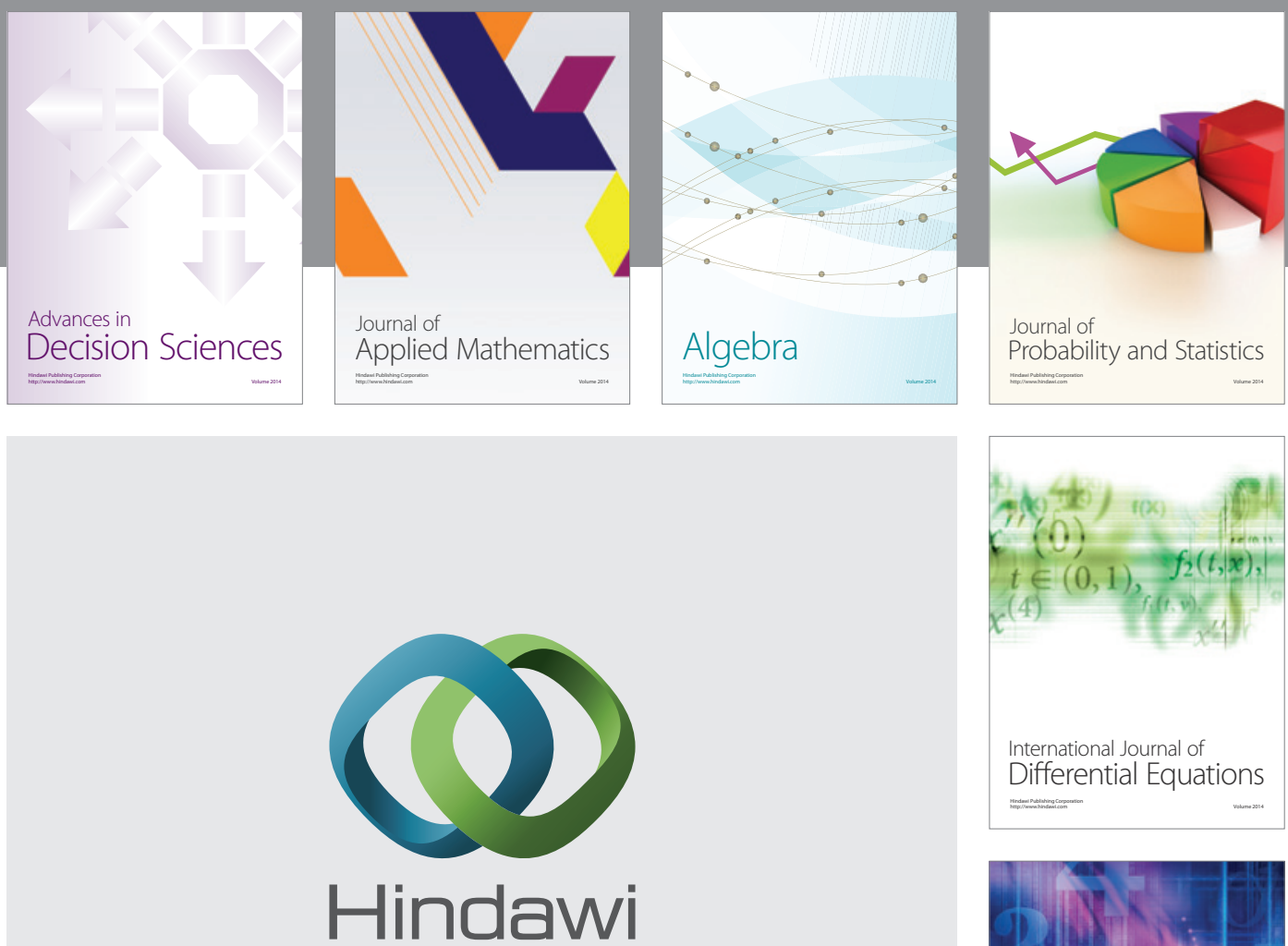

Submit your manuscripts at http://www.hindawi.com
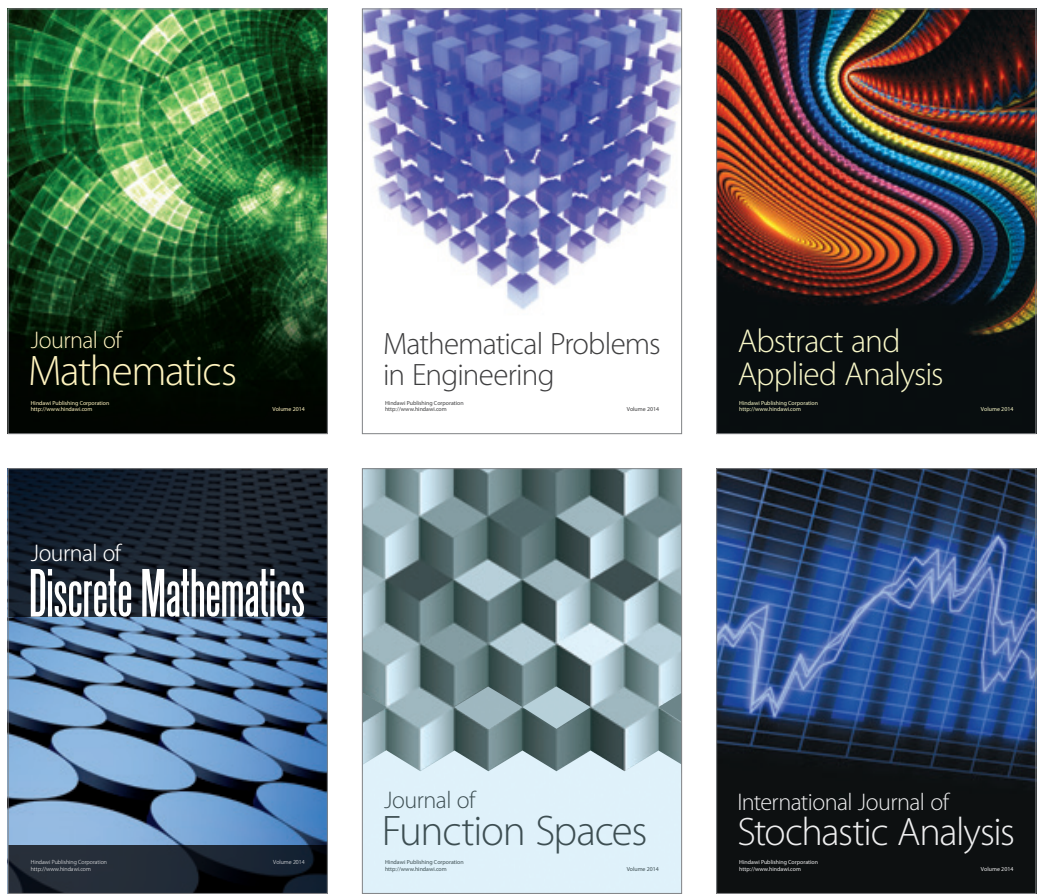

Journal of

Function Spaces

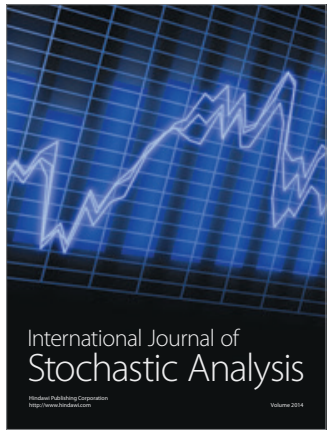

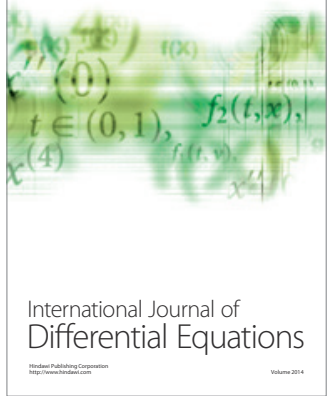
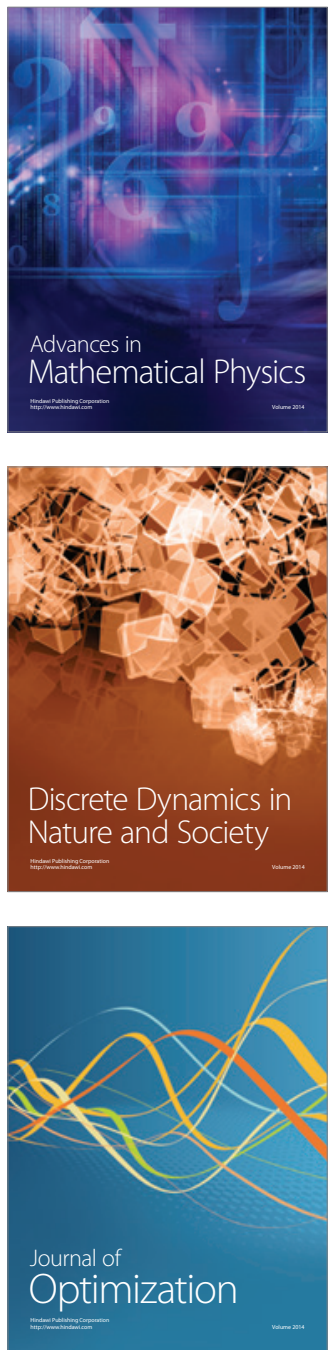